\title{
Iterative Refinement for Ill-Conditioned Linear Systems
}

\author{
Shin'ichi Oishi ${ }^{1,2}$, Takeshi Ogita ${ }^{2,1}$ and Siegfried M. Rump ${ }^{3}$
}

${ }^{1}$ Department of Computer Science, Faculty of Science and Engineering, Waseda University, 3-4-1 Okubo, Tokyo 169-8555 Japan

oishi@waseda.jp

${ }^{2}$ CREST, JST

${ }^{3}$ Institute for Reliable Computing, Hamburg University of Technology, Schwarzenbergstr. 9521071 Hamburg, Germany

\{rump@tu-harburg.de\}

\section{Introduction}

In this paper, we will consider the convergence of iterative refinement for a linear equation

$$
A v=b,
$$

where $A \in \mathbb{F}^{n \times n}$ and $b \in \mathbb{F}^{n}$. Here, $\mathbb{F}$ is a set of floating point numbers. Let $u$ be the unit round-off of the working precision and $\kappa(A)=\|A\|_{\infty}\left\|A^{-1}\right\|_{\infty}$ be the condition number of the problem. For well posed problems, i.e., in case of $u \kappa(A)<1$, it has been shown [1]-[4] that the iterative refinement improves the forward and backward errors of computed solutions provided that the residuals are evaluated by extended precision, in which the unit round off $\bar{u}$ is the order of $u^{2}$, before rounding back to the working precision. In this talk, we will treat ill-conditioned problems with

$$
1<u \kappa(A)<\infty .
$$

We can assume without loss of generality that for a certain positive integer $k$ the following is satisfied:

$$
u^{k} \kappa(A) \leqq \beta<1
$$

In Ref. [7], Rump has shown that for arbitrary ill-conditioned matrices $A$, we can have good approximate inverses $R_{1: k}$ satisfying $\left\|R_{1: k} A-I\right\|_{\infty} \leqq \alpha<1$. Here, $R_{1: k}$ is obtained as

$$
R_{1: k}=R_{1}+R_{2}+\cdots+R_{k}
$$

with $R_{i} \in \mathbb{F}^{n \times n}$. I is the $n$-dimensional unit matrix. In Ref. [5], we have partially clarified the mechanism of the convergence of Rump's method.

Let $A, B, C \in \mathbb{F}^{n \times n}$. We assume that we have an accurate matrix product calculation algorithm

$$
D_{1: k}=[A B-C]_{k}
$$

satisfying

$$
\left\|\sum_{i=1}^{k} D_{i}-(A B-C)\right\|_{\infty} \leqq c u^{k}\|A B-C\|_{\infty} .
$$

Such algorithms have been proposed, for instance, by the authors [6] and [8].

Now we propose the following iterative refinement algorithm:

$$
v^{\prime}=\left[v-R_{1: k}[A v-b]_{k}\right]_{1} .
$$

This scheme is a modification of the iterative refinement algorithm proposed in [9]. Put $r_{k}=$ $[A v-b]_{k}$ and let $\Phi(v)=\left[v-R_{1: k} r_{k}\right]_{1}$. Then, we can write

$$
v^{\prime}=\Phi(v) .
$$


The following holds:

$$
v^{\prime}=v-R_{1: k}\left[(A v-b)+e_{r}\right]+e_{m},
$$

where $e_{r}=r_{k}-(A v-b)$ and $e_{m} \in \mathbb{R}^{n}$ satisfying

$$
\left\|e_{r}\right\|_{\infty} \leqq c u^{k}\|A v-b\|_{\infty}, \quad\left\|e_{m}\right\|_{\infty} \leqq c u\left\|v-R_{1: k} r_{k}\right\|_{\infty} .
$$

In this paper, we will show the forward stability and backward stability of this iterative algorithm.

\section{References}

[1] G.B.Moler, "iterative refinement in floating point", J. Assoc. Comput. Mach., 14 316-321 (1967)

[2] R. D. Skeel, "Iterative refinement implies numerical stability for Gaussian elimination", Math. Comp., 35 817-832 (1980)

[3] N.J.Higham, "Iterative refinement for linear systems and LAPACK", IMA J. Numer. Anal. 17 495-509 (1997).

[4] M.Jankowsky and H.Woznlakowski, "Iterative refinement implies numerical stability", BIT 17 303-311 (1997).

[5] S. Oishi, K. Tanabe, T.Ogita, and S.M. Rump, "Convergence of Rump's method for inverting arbitrary ill-conditioned matrices", J. Comp. and Appl. Math, 205 533-544 (2007).

[6] T. Ogita, S. M. Rump and S. Oishi: "Accurate Sum and Dot Product", SIAM Journal on Scientific Computing, 26/6,1955-1988,(2005)

[7] S.M.Rump:" Approximate inverses of almost singular matrices still contain useful information", Technical Report 90.1, Faculty of Information and Communication Sciences, Hamburg University of Technology (1990).

[8] S. M. Rump, T. Ogita, and S. Oishi: Accurate Floating-Point Summation. 41 pages, submitted for publication, 2006. Preprint is available from

http://www.ti3.tu-harburg.de/publications/rump.

[9] T. Ohta, T. Ogita, S. M. Rump and S. Oishi: "A Method of Verified Numerical Computation for Ill-conditioned Linear System of Equations", Journal of JSIAM,15:3 (2005), pp. 269-287 in Japanese.

[10] S. M. Rump: A class of arbitrarily ill-conditioned floating-point matrices, SIAM J. Matrix Anal. Appl., 12:4 (1991), 645-653.

[11] J. D. Rigal and J. Gaches:" On the compativility of a given solution with the data of a linear equation", J. Assoc. Comput. Mach., 14 (1967), 543-548. 